Research Article

\title{
Complexity-Entropy Causality Plane Based on Return Intervals: A Useful Approach to Quantify the Aeroengine Gas Path Parameters
}

\author{
Keqiang Dong $\mathbb{D}$ and Linan Long \\ College of Science, Civil Aviation University of China, Tianjin 300300, China \\ Correspondence should be addressed to Keqiang Dong; 06121990@bjtu.edu.cn
}

Received 2 January 2018; Accepted 20 March 2018; Published 24 April 2018

Academic Editor: Oluwole D. Makinde

Copyright (C) 2018 Keqiang Dong and Linan Long. This is an open access article distributed under the Creative Commons Attribution License, which permits unrestricted use, distribution, and reproduction in any medium, provided the original work is properly cited.

\begin{abstract}
The complexity-entropy causality plane, as a powerful tool for discriminating Gaussian from non-Gaussian process, has been recently introduced to describe the complexity among time series. We propose to use this method to distinguish the stage of climbcruise-decline of aeroengine. Our empirical results demonstrate that this statistical physics approach is useful. Further, the return intervals based complexity-entropy causality plane is introduced to describe the complexity of aeroengine fuel flow time series. The results can infer that the cruise process has lowest complexity and the decline process has highest complexity.
\end{abstract}

\section{Introduction}

The understanding and analysis of aeroengine time series, especially the evolution of gas path system sequences, has been attracting the attention of mathematicians and physicists for many years. Doel and Urban modeled the gas path system by linear approaches [1,2], such as weighted-leastsquares [3], filtering approaches $[4,5]$, where the accuracy and reliability were limited. For improving calculation accuracy and reliability, nonlinear techniques, such as adaptive modeling [6, 7], neural networks [8-11], and genetic algorithms [12-15], were introduced to investigate the gas path system. The existence of autocorrelation between gas path system variables reveals the aeroengine efficiency because past observations can help to predict future variables. This question motivates the research on the subject, especially by aeroengine managers and analysts, trying to save aeroengine maintenance costs. For bearing fault detection, Liu has applied the detrended fluctuation analysis to analyze gas path system correlation [16]. Dong et al. have found that the exhaust gas temperature (EGT), the low-spool rotor speed (N1), the high-spool rotor speed (N2), and the fuel flow (FF) have greater correlation and cross-correlation than other observations, suggesting more predictability [17]. It was also shown that the EGT, N1, N2, and FF play an important role in understanding gas path system. Thus, the analysis of EGT, N1, N2, and FF seems to be more efficient than other observations.

Nowadays, it is clear that the design of aeroengine gas path system is a typical complex system, which involves many subcomponents. The computing ranges of aeroengine were chosen from the process of take-off-climb-cruise-declineapproach-land of aeroengine [18, 19]. To distinguish the process of take-off-climb-cruise-decline-approach-land of aeroengine depends on the airplane's altitude in previous analysis. In this paper, we propose to test the gas path system of aeroengine time series by employing a recently introduced statistical tool: the complexity-entropy causality plane. It is shown that this plane allows distinguishing cruise and decline process.

To make a distinction between climb, cruise, and decline process, the return intervals of aeroengine time series are proposed. The central quantities here are the time interval between successive events above (or below) some threshold Q. By studying the statistics of the return intervals for gas path system time series, one aims to find out the laws distinguishing the climb, cruise, and decline process throughout the flight. 
The organization of this paper is as follows. In the next section, we simply present the aeroengine gas path performance parameters employed in this paper. We show the complexity-entropy causality plane and empirical results in Section. In Section, we introduce a technique named return intervals based complexity-entropy causality plane enabling us to estimate the complexity characteristic for aeroengine gas path system. In particular, the ability to identify the complexity in gas path system is demonstrated. Finally, we draw some conclusions in Section.

\section{The Dataset}

In this paper, the complexity-entropy causality plane will be used to examine flight course of aeroengine. Here we make a brief description for gas path performance parameters, offered by Aircraft Maintenance and Engineering Corporation. In civil aviation flight management system, flight data are acquired from on-board flight data recorders, which are part of the Aircraft Condition Monitoring System (ACMS) such as Smart ACMS Recorder (SAR) and Quick Access Recorder (QAR) [20, 21]. The QAR data is more comprehensive where data includes an extensive list of flight parameters recorded at specific sampling intervals which are set by the manufacturer. Therefore, the QAR data is applied in this paper.

Previously, researches demonstrate that the parameters including EGT, N1, N2, and FF play an important role in understanding aeroengine system [17, 22]. For this reason, the parameters EGT, N1, N2, and FF are usually selected to amplify the study of aeroengine gas path system. Here, the parameter FF is selected as an example in this paper.

\section{Complexity-Entropy Causality Plane and Empirical Results}

3.1. Complexity-Entropy Causality Plane Method. For measuring the information content of aeroengine system, a typical method is to evaluate probability distribution function, describing the distribution of some measurable or observable property. Therefore, the Shannon entropy, which can be of great help when analyzing aeroengine system data since it captures the uncertainty and disorder of the time series without imposing any limitations on the theoretical probability distribution [23-25], is used as a first natural approach. For a time series $X=\left\{x_{i}: i=1,2, \ldots, n\right\}$ with probability distribution $P=\left\{p\left(x_{i}\right): i=1,2, \ldots, n\right\}$, the Shannon entropy is given by [26-28]

$$
H(P)=-\sum_{x_{i}} p\left(x_{i}\right) \log p\left(x_{i}\right) .
$$

The Shannon entropy $H(P)=0$, if the time series $X$ is certain which of the possible outcomes $x_{i}$ whose probabilities are given by $p\left(x_{i}\right)$ will actually take place. The Shannon entropy should be maximal, if all the outcomes are equally likely (uncertainty is highest when all possible events are equiprobable).

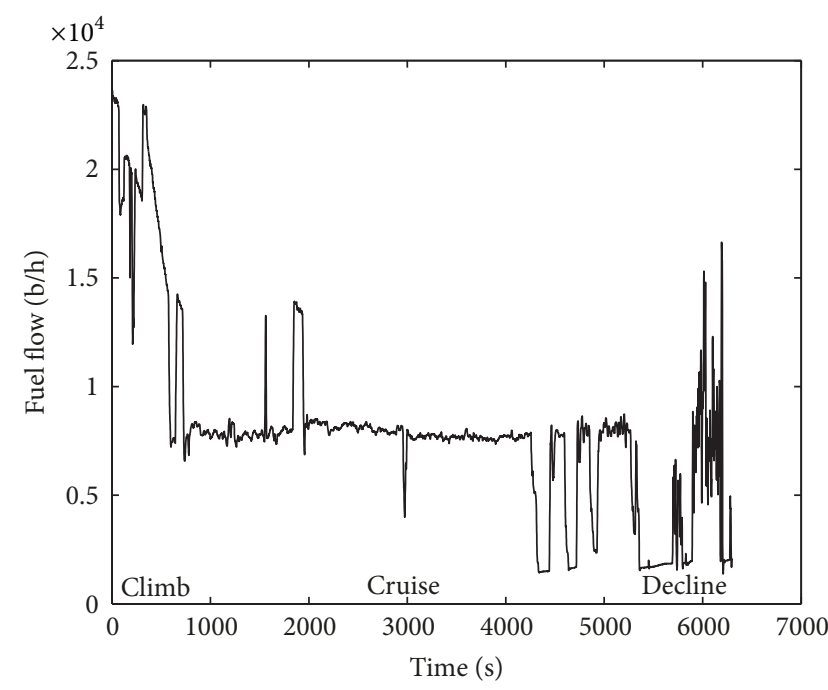

FIGURE 1: The process of climb-cruise-decline of aeroengine gas path system for FF time series.

For differentiating different degrees of periodicity and chaos, nevertheless, Lamberti et al. [29] proposed a statistical complexity measure (SCM) method. This method offers significant additional information regarding the peculiarities of the underlying probability distribution, not detected by the entropy. It is defined as follows:

$$
C_{J S}(P)=Q_{J}\left(P, P_{e}\right) H_{S}(P),
$$

where $H_{S}(P)=H(P) / S_{\max }$ is the normalized Shannon entropy, with $S_{\max }=H\left(P_{e}\right)=\ln N,\left(0 \leq H_{S} \leq 1\right)$, and $P_{e}=\{1 / N, \ldots, 1 / N\}$.

$Q_{J}\left(P, P_{e}\right)=Q_{0} J\left(P, P_{e}\right)$ with $J\left(P, P_{e}\right)=H\left(P+P_{e}\right) / 2-$ $H(P) / 2-H\left(P_{e}\right) / 2$ and $Q_{J}$ is defined in terms of the extensive Jensen-Shannon divergence, and $Q_{0}$ is a normalization constant, equal to the inverse of maximum possible value of $J\left(P, P_{e}\right)$.

The diagram of $C_{I S}$ versus $H_{S}(P)$ is the complexityentropy causality plane defined in $[23,30]$. Statistical complexity measure was recently shown to be necessary because it captures the property of organization [31]. SCM has been successfully used to research changes in system dynamics originated by modifications of some characteristic parameters [32-34].

3.2. Complexity-Entropy Causality Plane Results. In this section, we analyze the gas path system fuel flow $(\mathrm{FF})$ parameters for 10 aeroengines. The data of the FF variables for climbcruise-decline process are presented in Figure 1. We employ gas path parameters over climb-cruise-decline process, where the cruise process has lowest complexity (see Figure 1). In order to make comparisons, all aeroengines gas path parameters are studied for the same time length.

In Figure 2, we plot the cruise and decline of the different aeroengines for FF time series in the complexity-entropy causality plane. Observe that the cruise process has higher entropy (lower complexity) than decline ones. Therefore, the former is closer to the ideal point 


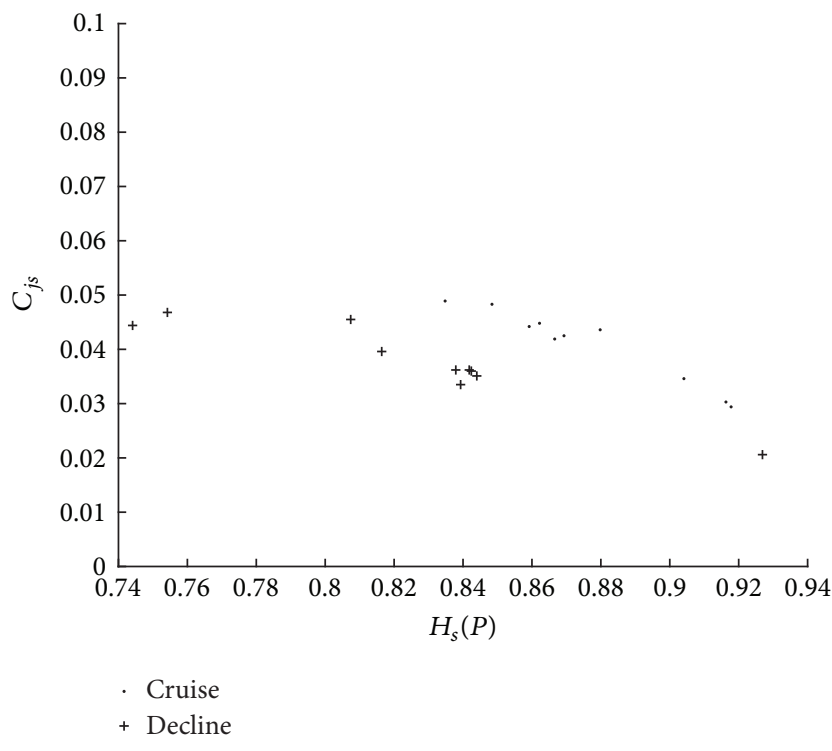

FIGURE 2: The complexity-entropy causality plane of the cruise and decline for the different aeroengines FF time series.

\section{Return Interval Based Complexity-Entropy Causality Plane and Empirical Results}

4.1. Return Interval Based Complexity-Entropy Causality Plane Method. The understanding of the fluctuation of FF is of great importance in aeroengine fields. One of the central quantities here is the time interval between successive flows above (or below) some threshold $Q$, sometimes referred to as reoccurrence times or return intervals. Inspired by [2426], we give the time interval for a time series $X=\left\{x_{i}: i=\right.$ $1,2, \ldots, n\}$ as follows.

Step 1. Describe the first-order difference $\Delta^{(1)} X=\left\{\Delta x_{i}: i=\right.$ $1,2, \ldots, n\}$

$$
\Delta x_{i}=x_{i}-x_{i-1}
$$

Step 2. Calculate the time interval between successive sequences above threshold 0 for the first-order difference $\Delta^{(1)} X$, that is, the time interval between fuel flows that start increasing.

Step 3. Compute $C_{J S}$ and $H_{S}(P)$ for time interval sequences, and then plot $C_{J S}$ versus $H_{S}(P)$.

4.2. Return Interval Based Complexity-Entropy Causality Plane Results. Next, we study the way the return intervals are arranged in time. Figure 3 shows the complexity-entropy causality plane of the cruise and decline for the different aeroengines FF time series. Obviously, the higher entropy in cruise process has been induced by the return interval based complexity-entropy causality plane, compared with Figure 2. Accordingly, the return interval time series are sensitive indicators for original series.

In order to gain a deeper insight, we analyze the complexity of climb-cruise-decline for return interval based

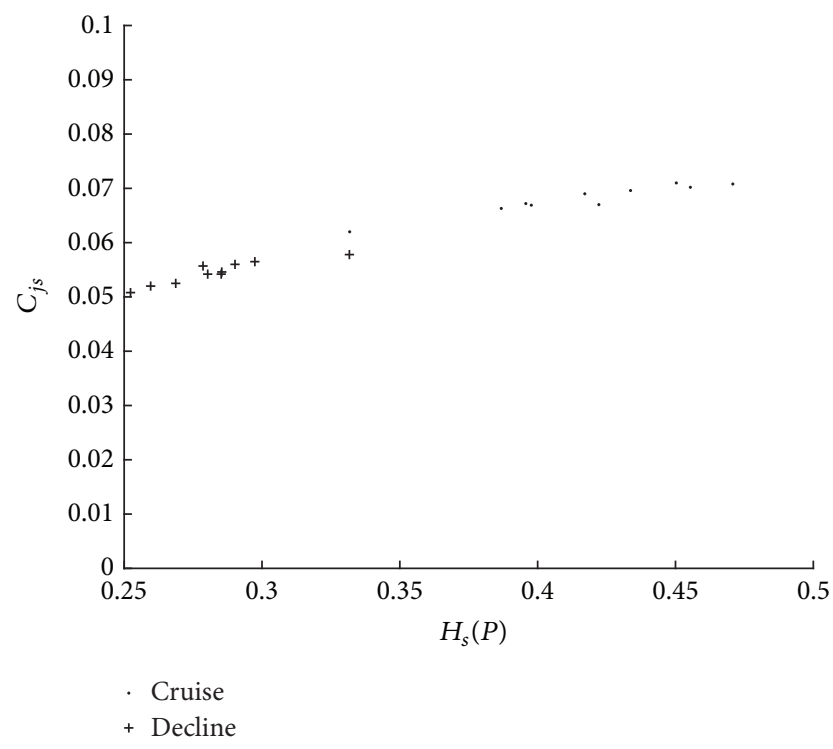

FIGURE 3: The complexity-entropy causality plane of the cruise and decline for the different aeroengines FF return intervals time series.

aeroengines FF time series in Figure 4. Figure 4(a) is the complexity-entropy causality plane of climb and cruise process for the different aeroengines FF time series and Figure 4(b) gives the climb and decline process for the different aeroengines FF time series.

Figure 1 shows that the cruise process has lowest complexity and the decline process has highest complexity. Thus we expect that the result of return intervals will show a similar behavior, too. This is shown in Figure 4, where, for climb, cruise, and decline values, the cruise process has highest entropy, and the decline process has lowest entropy. Furthermore, this return interval based method provides an effective method to distinguish the stage of climb-cruisedecline of aeroengine. The agreement between observed data and return interval based model is striking.

\section{Conclusion}

In this paper, we consider complexity-entropy causality plane method to understand the complexity characteristics in aeroengine gas path system firstly. The technique has been implemented on the fuel flow time series. Our empirical results demonstrate that the cruise process has lower complexity than decline ones.

And then, by constructing the return intervals based complexity-entropy causality plane method to fuel flow parameters, we obtain the similar complexity characteristics exhibited by $C_{J S}$ versus $H_{S}(P)$ implying the higher entropy of fuel flow time series for the cruise and decline process.

To capture the degree of climb-cruise-decline of aeroengine in detail, we apply return intervals based complexityentropy causality plane to research the complexity between climb-cruise-decline processes for aeroengine gas path systems. The results not only distinguish the stage of climbcruise-decline of aeroengine, but also infer that the cruise 


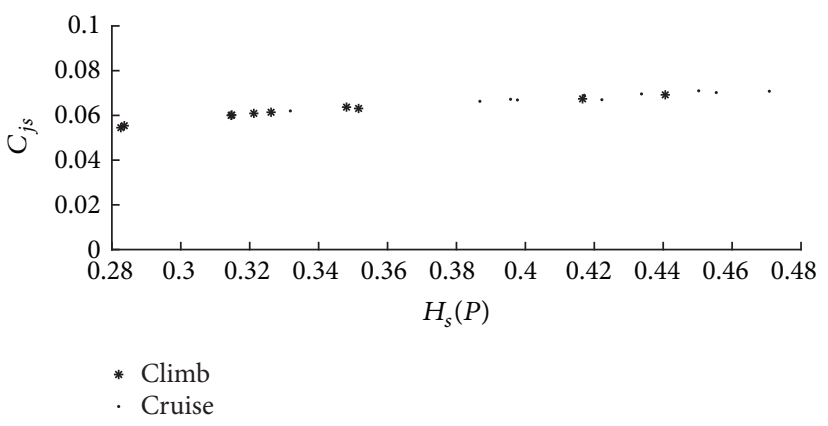

(a)

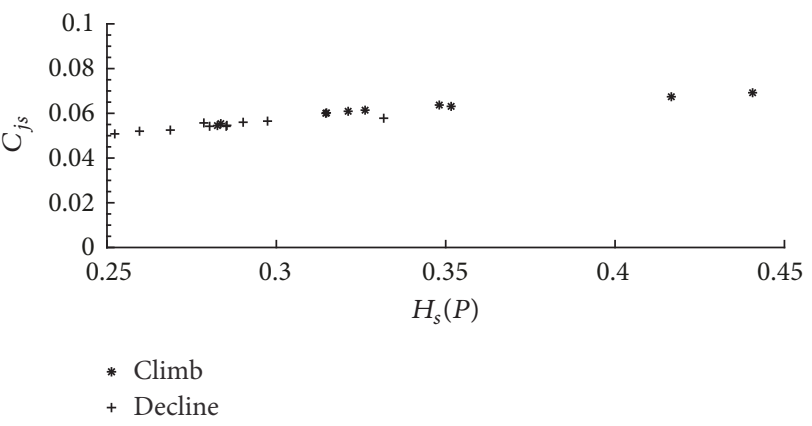

(b)

FIGURE 4: The complexity-entropy causality plane of (a) the climb and cruise and (b) climb and decline for the different aeroengines FF return intervals time series.

process has lowest complexity and the decline process has highest complexity.

\section{Conflicts of Interest}

The authors declare that there are no conflicts of interest regarding the publication of this article.

\section{Acknowledgments}

The financial support from the funds of the National Natural Science Foundation of China under Grant no. 61401467 and the Fundamental Research Funds for the Central Universities under Grant no. 3122017084 is gratefully acknowledged.

\section{References}

[1] D. L. Doel, "TEMPER-a gas-path analysis tool for commercial jet engines," in Proceedings of the ASME 1992 International Gas Turbine and Aeroengine Congress and Exposition, GT 1992, June 1992.

[2] L. A. Urban, "Gas path analysis applied to turbine engine condition monitoring," in Proceedings of the 8th Joint Propulsion Specialist Conference, 1972.

[3] D. L. Doel, "An assessment of weighted-least-squares based gas path analysis," in Proceedings of the ASME 1993 internatioal gas turbine and aeroengine congress and exposition, Cincinnati, $\mathrm{OH}$, USA, May 1993.

[4] A. J. Volponi, H. DePold, R. Ganguli, and C. Daguang, "The use of kalman filter and neural network methodologies in gas turbine performance diagnostics: a comparative study," Journal of Engineering for Gas Turbines and Power, vol. 125, no. 4, pp. 917-924, 2003.

[5] D. Simon, "A comparison of filtering approaches for aircraft engine health estimation," Aerospace Science and Technology, vol. 12, no. 4, pp. 276-284, 2008.

[6] A. Stamatis, K. Mathioudakis, and K. D. Papailiou, "Adaptive simulation of gas turbine performance," in Proceedings of the Preprint - American Society of Mechanical Engineers, June 1989.

[7] B. Lambiris, K. Mathioudakis, A. Stamatis, and K. Papailiou, "Adaptive modeling of jet engine performance with application to condition monitoring," Journal of Propulsion and Power, vol. 10, no. 6, pp. 890-896, 1994.
[8] C. Romessis and K. Mathioudakis, "Setting up of a probabilistic neural network for sensor fault detection including operation with component faults," in Proceedings of the ASME TURBO Expo 2002:Controls, Diagnostics, and Instrumentation, Cycle Innovations, Marine, Oil and Gas Applications, pp. 101-108, June 2002.

[9] M. J. Embrechts, A. L. Schweizerhof, M. Bushman, and M. H. Sabatella, "Neural network modeling of turbofan parameters," in Proceedings of the ASME Turbo Expo 2000: Power for Land, Sea, and Air, GT 2000, May 2000.

[10] M. F. Abdul Ghafir, Y. G. Li, and L. Wang, "Creep life prediction for aero gas turbine hot section component using artificial neural networks," Journal of Engineering for Gas Turbines and Power, vol. 136, no. 3, Article ID 031504, 2014.

[11] X. Pu, S. Liu, H. Jiang, and D. Yu, "Sparse bayesian learning for gas path diagnostics," Journal of Engineering for Gas Turbines and Power, vol. 135, no. 7, Article ID 071601, 2013.

[12] M. Zedda and R. Singh, "Gas turbine engineand sensor diagnostics," ISABE 99-7238, 1999.

[13] S. Sampath, S. O. T. Ogaji, Y. G. Li, and R. Singh, "Fault diagnosis of a two spool turbo-fan engine using transient data: A genetic algorithm approach," in Proceedings of the 2003 ASME Turbo Expo, pp. 351-359, June 2003.

[14] A. Gulati, M. Zedda, and R. Singh, "Gas turbine engine and sensor multiple operating point analysis using optimization techniques," in Proceedings of the 36th AIAA/ASME/SAE/ASEE Joint Propulsion Conference and Exhibit, July 2000.

[15] M. Wallin, T. Gronstedt, and T. Grōnstedt, "A comparative study of genetic algorithms and gradient methods for RM12 turbofan engine diagnostics and performance estimation," GT200453591, 2004.

[16] J. Liu, "Detrended fluctuation analysis of vibration signals for bearing fault detection," in Procsedings of the IEEE Conference on Prognostics and Health Management, pp. 1-5, Denver, Colorado, USA, June 2011.

[17] K. Dong, H. Zhang, and Y. Gao, "Dynamical mechanism in aero-engine gas path system using minimum spanning tree and detrended cross-correlation analysis," Physica A: Statistical Mechanics and its Applications, vol. 465, pp. 363-369, 2017.

[18] C.-W. Fei and G.-C. Bai, "Distributed collaborative probabilistic design for turbine blade-tip radial running clearance using support vector machine of regression," Mechanical Systems and Signal Processing, vol. 49, pp. 196-208, 2014. 
[19] B. L. Scott and M. S. Bruce, "Turbine Engine Clearance Control Systems: Current Practices and Future Directions," NASA/TM 2002-211794, 2002.

[20] H. Haverdings and P. W. Chan, "Quick Access Recorder (QAR) data analysis software for windshear and turbulence studies," in Proceedings of the 1st AIAA Atmospheric and Space Environments Conference, San Antonio, Tex, USA, June 2009.

[21] T. McDade, "Advances in flight data acquisition and management systems," in Proceedings of the 17th DASC. AIAA/IEEE/ SAE Digital Avionics Systems Conference. Proceedings, pp. F12/1F12/8, Bellevue, WA, USA, November 1998.

[22] K. Dong, J. Fan, and Y. Gao, "Cross-correlations and structures of aero-engine gas path system based on DCCA coefficient and rooted tree," Fluctuation and Noise Letters, vol. 14, no. 2, Article ID 1550014, 2015.

[23] Z. Luciano, Z. Massimiliano, and M. T. Benjamin, "Complexityentropy causality plane: A useful approach to quantify the stock market inefficiency," Physica A: Statistical Mechanics and its Applications, vol. 389, no. 9, pp. 1891-1901, 2010.

[24] G. A. Darbellay and D. Wuertz, "The entropy as a tool for analysing statistical dependences in financial time series," Physica A: Statistical Mechanics and its Applications, vol. 287, no. 3-4, pp. 429-439, 2000.

[25] S. R. Bentes, R. Menezes, and D. A. Mendes, "Long memory and volatility clustering: is the empirical evidence consistent across stock markets?" Physica A: Statistical Mechanics and its Applications, vol. 387, no. 15, pp. 3826-3830, 2008.

[26] C. E. Shannon, "A mathematical theory of communication," Bell Labs Technical Journal, vol. 27, pp. 379-423, 623-656, 1948.

[27] Y. Teng and P. Shang, "Transfer entropy coefficient: Quantifying level of information flow between financial time series," Physica A: Statistical Mechanics and its Applications, vol. 469, pp. 60-70, 2017.

[28] G.-J. Wang, C. Xie, Y.-J. Chen, and S. Chen, "Statistical properties of the foreign exchange network at different time scales: evidence from detrended cross-correlation coefficient and minimum spanning tree," Entropy. An International and Interdisciplinary Journal of Entropy and Information Studies, vol. 15, no. 5, pp. 1643-1662, 2013.

[29] P. W. Lamberti, M. T. Martin, A. Plastino, and O. A. Rosso, "Intensive entropic non-triviality measure," Physica A: Statistical Mechanics and its Applications, vol. 334, no. 1-2, pp. 119-131, 2004.

[30] O. A. Rosso, H. A. Larrondo, M. T. Martin, A. Plastino, and M. A. Fuentes, "Distinguishing noise from chaos," Physical Review Letters, vol. 99, no. 15, Article ID 154102, 2007.

[31] D. P. Feldman, C. S. McTague, and J. . Crutchfield, "The organization of intrinsic computation: complexity-entropy diagrams and the diversity of natural information processing," Chaos: An Interdisciplinary Journal of Nonlinear Science, vol. 18, no. 4, Article ID 043106, pp. 148-201, 2008.

[32] X. Calbet and R. López-Ruiz, "Tendency towards maximum complexity in a nonequilibrium isolated system," Physical Review E: Statistical Physics, Plasmas, Fluids, and Related Interdisciplinary Topics, vol. 63, no. 6, 2001.

[33] O. A. Rosso, M. T. Martin, A. Figliola, K. Keller, and A. Plastino, "EEG analysis using wavelet-based information tools," Journal of Neuroscience Methods, vol. 153, no. 2, pp. 163-182, 2006.

[34] O. A. Rosso, H. Craig, and P. Moscato, "Shakespeare and other English Renaissance authors as characterized by Information Theory complexity quantifiers," Physica A: Statistical Mechanics and its Applications, vol. 388, no. 6, pp. 916-926, 2009. 


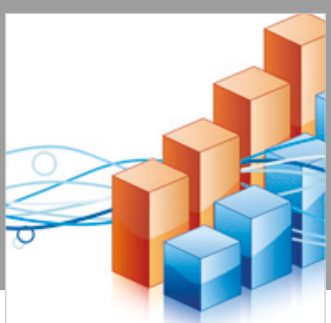

Advances in

Operations Research

\section{-n-m}
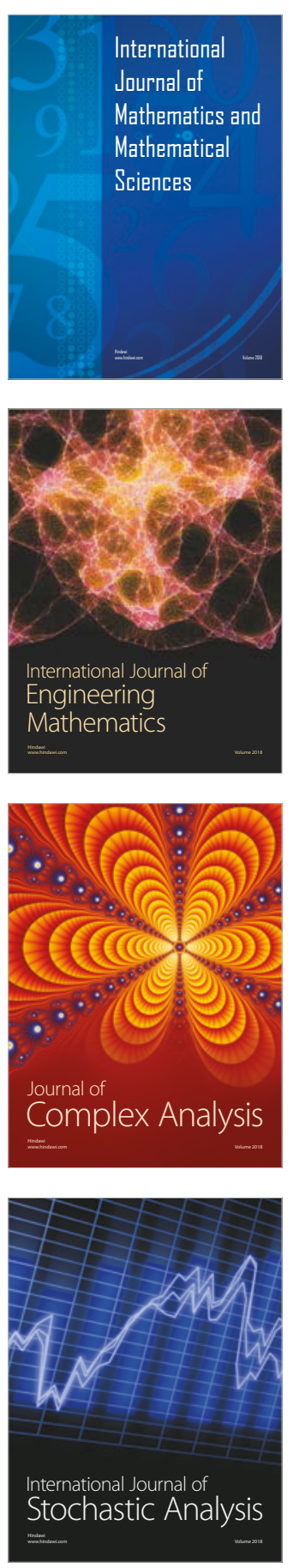
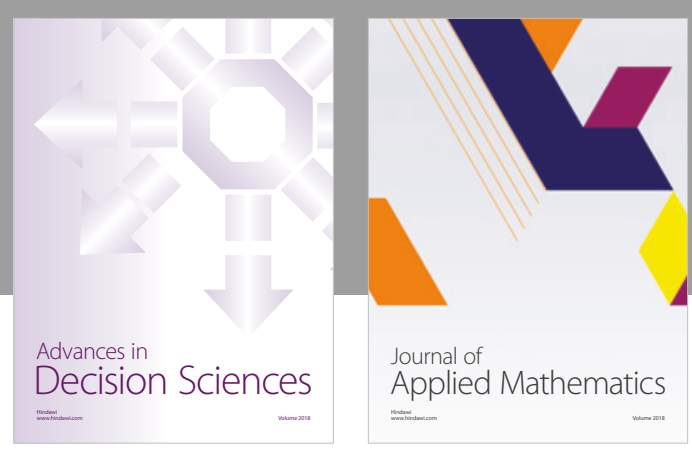

Journal of

Applied Mathematics
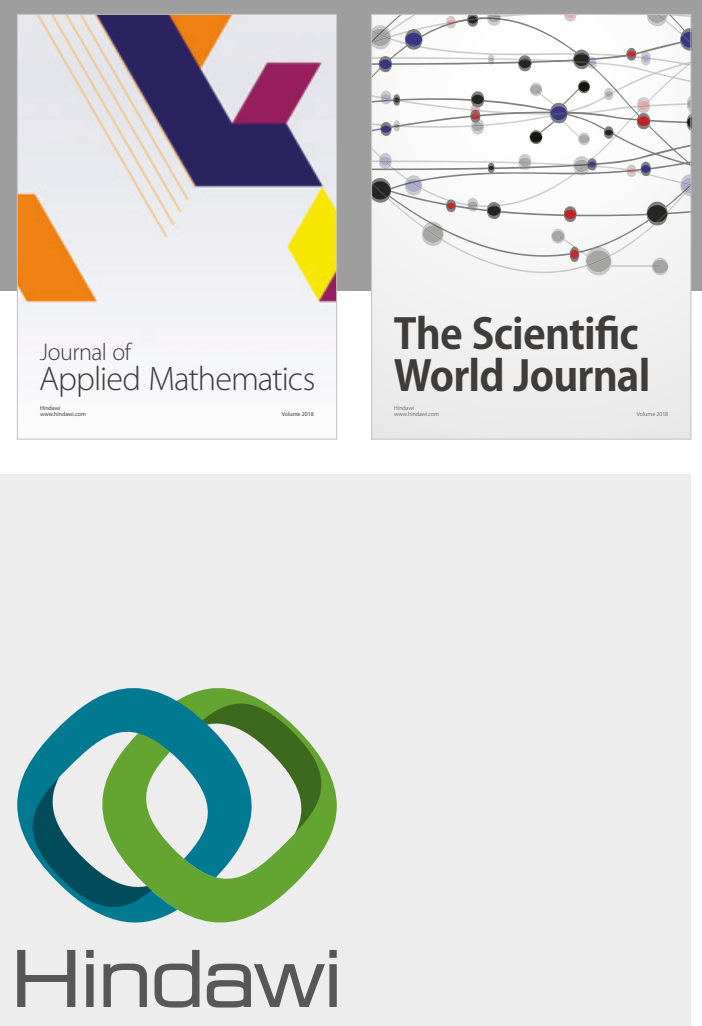

Submit your manuscripts at

www.hindawi.com

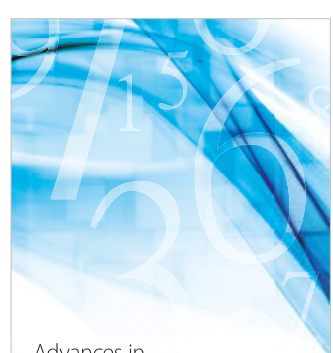

Advances in
Numerical Analysis
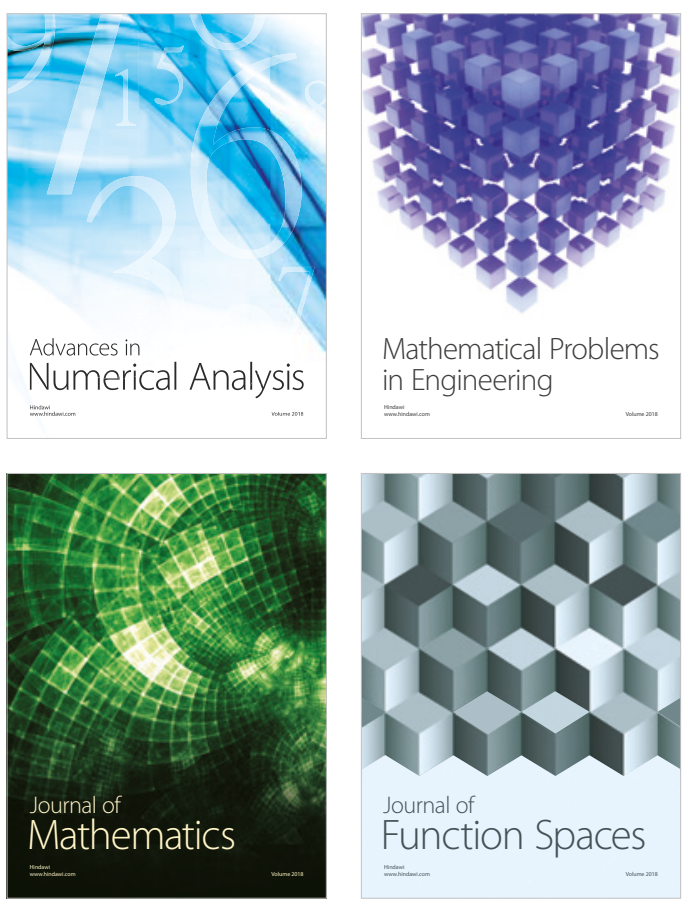

Mathematical Problems in Engineering

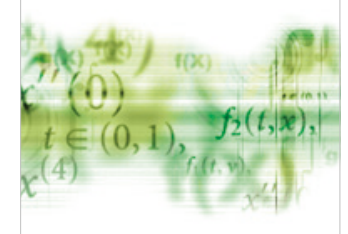

International Journal of

Differential Equations

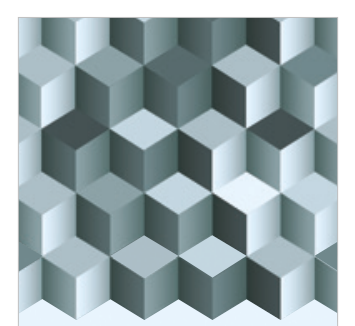

Journal of

Function Spaces
The Scientific

World Journal

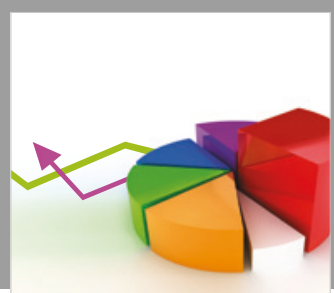

Journal of

Probability and Statistics
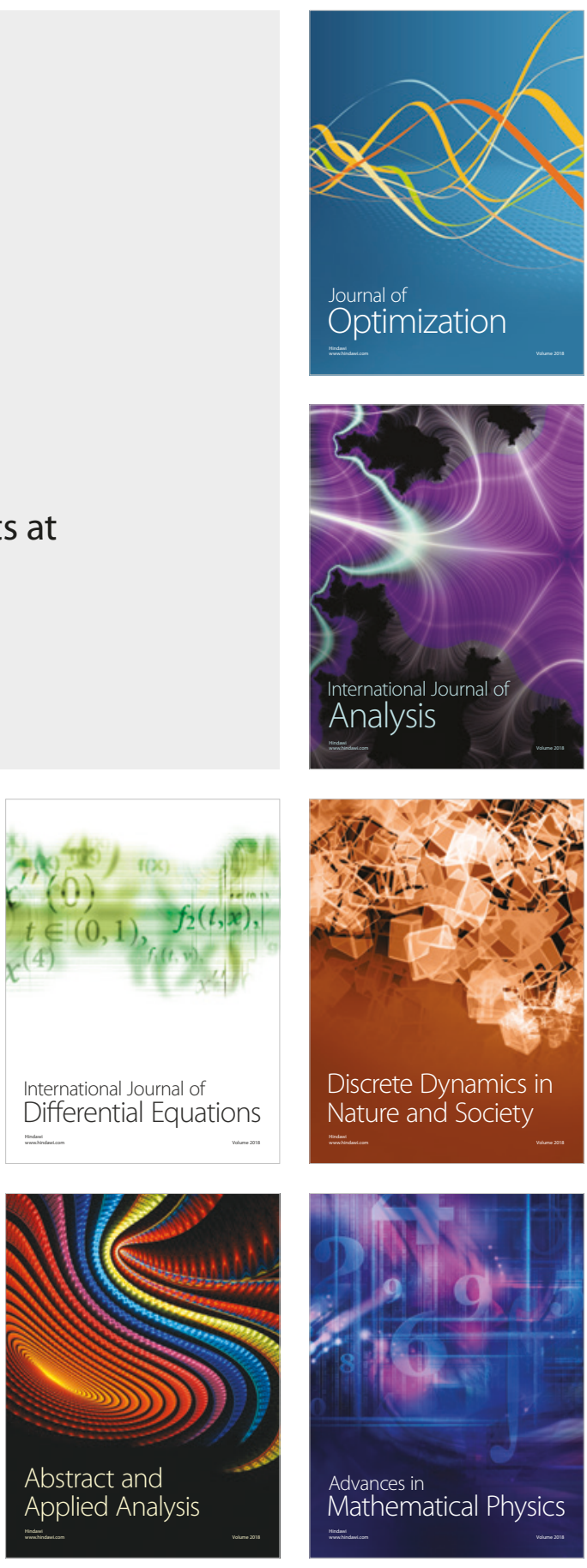